Document downloaded from:

http://hdl.handle.net/10251/154805

This paper must be cited as:

Vendrell-Criado, V.; González-Bello, C.; Miranda Alonso, MÁ.; Jiménez Molero, MC. (2018). A combined photophysical and computational study on the binding of mycophenolate mofetil and its major metabolite to transport proteins. Spectrochimica Acta Part A Molecular and Biomolecular Spectroscopy. 199:308-314. https://doi.org/10.1016/j.saa.2018.03.064

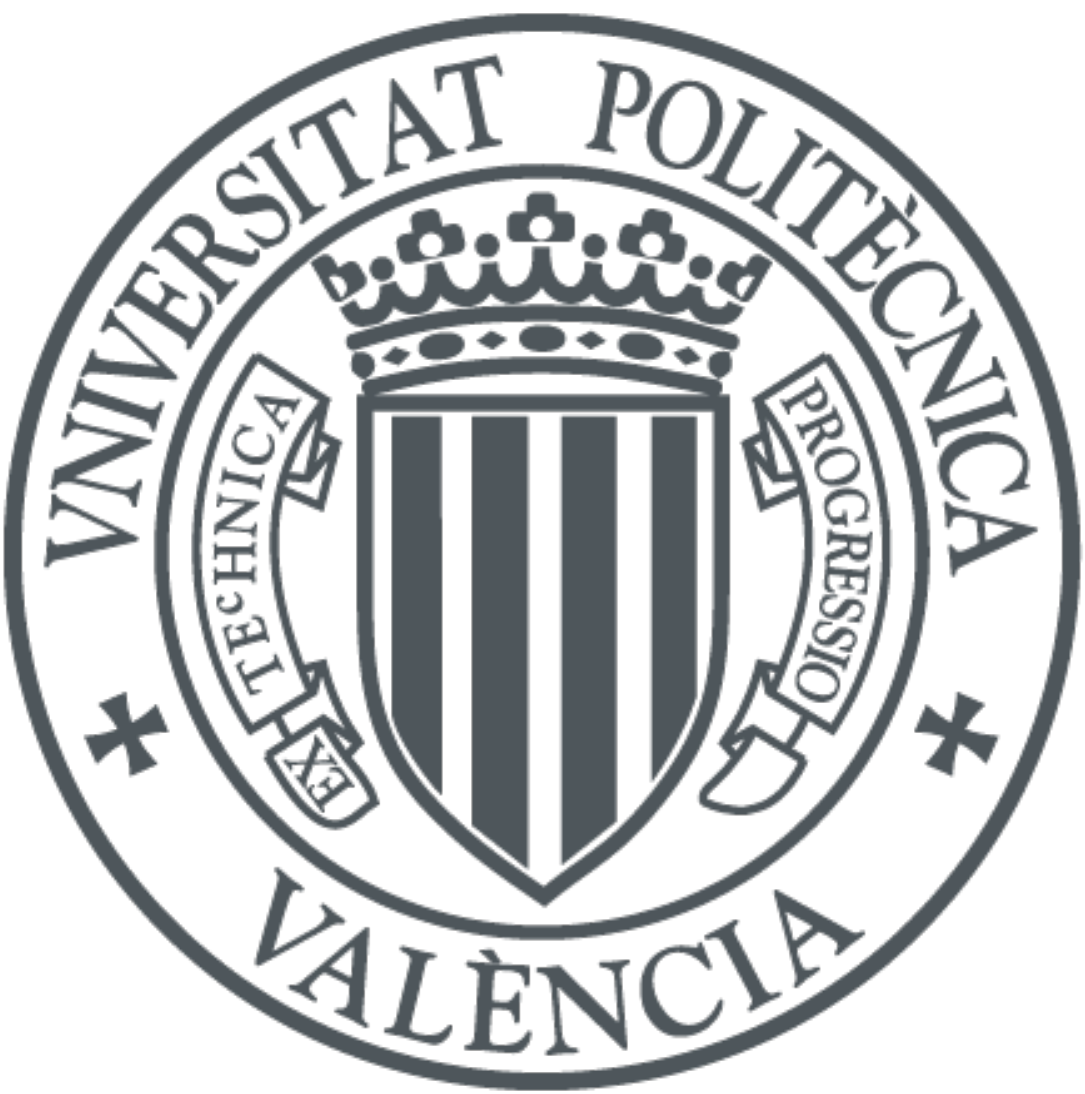

The final publication is available at

https://doi.org/10.1016/j.saa.2018.03.064

Copyright Elsevier

Additional Information 


\section{Graphical Abstract}

\section{A Combined Photophysical and}

Computational Study on the Binding of

Mycophenolate Mofetil and its Major

Metabolite to Transport Proteins

Victoria Vendrell-Criado, Concepción González-Bello, Miguel A. Miranda* and M. Consuelo Jiménez*

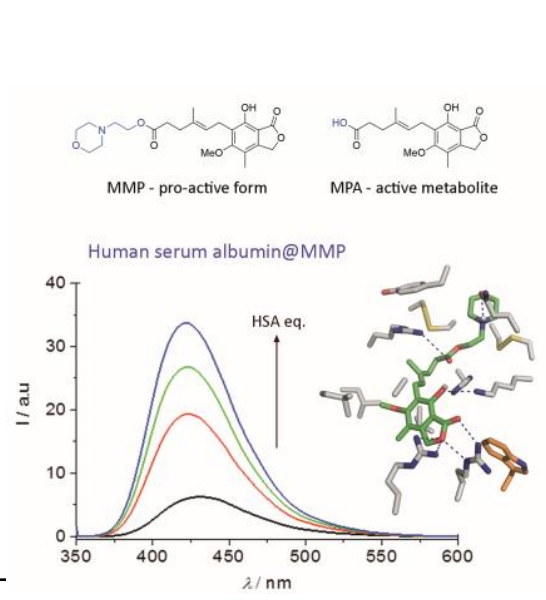

Leave this area blank for abstract info.

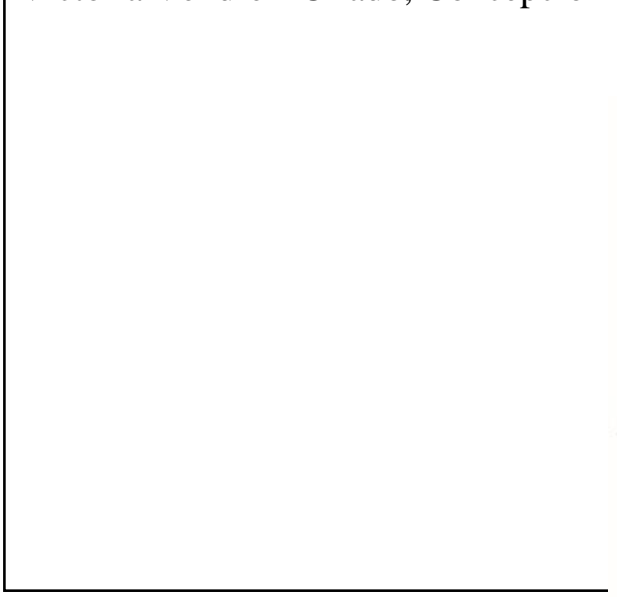




\title{
A Combined Photophysical and Computational Study on the Binding of Mycophenolate Mofetil and its Major Metabolite to Transport Proteins
}

\author{
Victoria Vendrell-Criado ${ }^{\mathrm{a}}$, Concepción González-Bello ${ }^{\mathrm{b}}$, Miguel A. Miranda ${ }^{\mathrm{a}, *}$ and M. Consuelo Jiménez ${ }^{\mathrm{a}, *}$ \\ ${ }^{a}$ Departamento de Química/Instituto de Tecnología Química UPV-CSIC, Universitat Politècnica de València, Camino de Vera s/n, 46022, Valencia, Spain \\ ${ }^{b}$ Centro Singular de Investigación en Química Biolóxica e Materiais Moleculares (CIQUS), Departamento de Química Orgánica, Universidade de Santiago de \\ Compostela, Jenaro de la Fuente s/n, 15782 Santiago de Compostela, Spain
}

\section{ARTICLE INFO}

Article history:

Received

Revised

Accepted

Available online

\section{Keywords:}

drug-protein binding

docking

fluorescence

human serum albumin

human $\alpha_{1}$-acid glycoprotein

\section{ABSTRACT}

Binding of the inmunodrepresive agent mycophenolate mofetil (MMP) and its pharmacologically active metabolite mycophenolic acid (MPA) to human serum albumin (HSA) and $\alpha_{1}$-acid glycoprotein (HAAG) has been investigated by an integrated approach involving selective excitation of the drug fluorophore, following their UV-A triggered fluorescence and docking studies. The formation of the protein/ligand complexes was evidenced by a dramatic enhancement of the fluorescence intensity and a hypsochromic shift of the emission band. In HSA, competitive studies using oleic acid as site I probe revealed site I as the main binding site of the ligands. Binding constants revealed that the affinity of the active metabolite by HSA is four-fold higher than its proactive form. Moreover, the affinity of MMP by HSA is three-fold higher than by HAAG. Docking studies revealed significant molecular binding differences in the binding of MMP and MPA to sub-domain IIA of HSA (site 1). For MPA, the aromatic moiety would be in close contact to Trp214 with the flexible chain pointing to the other end of the subdomain; on the contrary, for MMP, the carboxylate group of the chain would be fixed nearby Trp214 through electrostatic interactions with residues Arg218 and Arg222.

2009 Elsevier Ltd. All rights reserved.

\section{Introduction}

The binding of drugs to plasma proteins determines their biodistribution, elimination and therapeutic or toxic effects. It is generally accepted that only the unbound form of a drug interacts with the receptor, to produce pharmacological effects. This principle is the basis for understanding the pharmacokinetic behavior and the pharmacological activity of those drugs that are fast and extensively bound to plasma proteins at therapeutic concentrations [1].

Among plasma proteins, human serum albumin (HSA) and human $\alpha_{1}$-acid glycoprotein (HAAG) play a crucial role as drug carriers [2]. Thus, HSA is a globular protein of 585 amino acids, which accounts for about $60 \%$ of the total protein amount in blood serum. It has an exceptional binding capacity for a wide range of endogenous and exogenous ligands, being an important determinant of the pharmacokinetic behavior of many drugs. Its structure is well-known, containing three homologous domains assembled to form a heart-shaped macromolecule. Each domain contains two subdomains with common structural motifs. The principal regions of ligand binding to HSA are located in hydrophobic cavities of subdomains IIA and IIIA [3]. Concerning HAAG, it is less abundant than HSA, although its serum concentration increases in response to a systemic tissue injury, inflammation, or infection. HAAG contains 183 amino acids, two disulfide bridges, and the carbohydrate content represents $45 \%$ of the molecular weight attached in the form of five to six highly sialylated complex-type- $N$-linked glycans. This protein has the ability to bind and carry mainly basic and neutral lipophilic ligands from endogenous and exogenous origin and has one main binding site, which is large and flexible [4]. Most acidic, neutral, and basic drugs bind to HSA, and a few basic drugs bind almost exclusively to HAAG [5].

Mycophenolate mofetil (MMP) is an inmunodepresive agent, prescribed to prevent acute rejection of transplanted organs [6]. It is a prodrug that undergoes rapid hydrolysis by esterases in liver and kidney, to afford the pharmacologically active metabolite form mycophenolic acid (MPA) [7]; the latter behaves as selective and reversible inhibitor or inosine monophosphate dehydrogenase, acting on $\mathrm{T}$ and $\mathrm{B}$ lymphocytes. Other uses of 
MMP are as antimicrobial, anti-inflammatory, antifibrotic, proapoptotic, antiangiogenic, anticancerous or antioxidant [8].<smiles>[R]OC(=O)CC/C(C)=C/Cc1c(O)c2c(c(C)c1OC)COC2=O</smiles>

Chemical structures of MMP and MPA

It is known that MPA is mostly bound to HSA (98-99\%), and that its pharmacological activity is linked to the concentration of unbound drug. Factors such as hypoalbuminemia influence the protein binding of MPA, altering the free concentration in vivo. In addition, MPA-protein binding is relevant in renal transplantation and its insufficiency [9].

In this context, the binding of MPA to HSA has been explored in the past by following the fluorescence quenching of the Trp residue of HSA in the presence of increasing amounts of MPA. However, it is not possible to excite Trp selectively, due to spectral overlap with MPA, so fluorescence quenching can be masked by filtering effects, resulting in misleading interpretations $[8 \mathrm{a}, 10]$. The problems are circumvented in the present work by interrogating the binding of MMP and its major metabolite MPA to transport proteins (HSA and HAAG), which allows selective excitation of the drug fluorophore, following their UV-A triggered fluorescence. The results are consistent with those obtained by means of computational studies.

\section{Results and Discussion}

In this section, the photophysical properties in homogenous drug solutions, corresponding to the free forms, are presented first. This is followed by the results obtained with the bound forms, using HSA and HAAG as host proteins. Variations observed in the steady-state and time-resolved emission experiments, specifically the fluorescence quantum yields and lifetimes, can be associated with the binding process. This, in combination with the results obtained using displacement probes, is exploited to provide key information on the nature of the binding site and the magnitude of the binding constant. Finally, computational studies are presented that explain the experimental results.

\section{Photophysical properties of MMP and MPA in PBS}

The absorption spectrum of MMP in PBS (phosphate buffered saline solution, $\mathrm{pH}=7.4$ ) is shown in Figure 1A (black trace). Two bands can be observed above $280 \mathrm{~nm}$, in the UV-A and UV$B$ regions, with maxima at $\lambda=340 \mathrm{~nm}$ and $310 \mathrm{~nm}$, respectively; they are ascribed to phenolate and phenol MMP species, according to previous reports on the absorption spectra at different $\mathrm{pH}$ values [11]. As expected, the absorption spectrum of MPA is very similar (Figure 1B), since both MMP and MPA contain the same active chromophore.

The fluorescence emission and excitation spectra of MMP and MPA were also recorded in PBS (Figures 2A and 2B, respectively). For both compounds, the emission band exhibited a maximum at $\lambda=432 \mathrm{~nm}$, which did not depend on the excitation wavelength (310 or $340 \mathrm{~nm}$ ); such emission is attributed to deprotonated phenolate singlet excited state, according to the excitation spectrum with maximum at $\lambda=340 \mathrm{~nm}$. From the intersection between normalized excitation and emission spectra (values between 380 and $382 \mathrm{~nm}$ in case of MMF and 377 and
$381 \mathrm{~nm}$ in case of MPA), the singlet energy of the phenolate species is $c a .75 \mathrm{kcal} / \mathrm{mol}$.
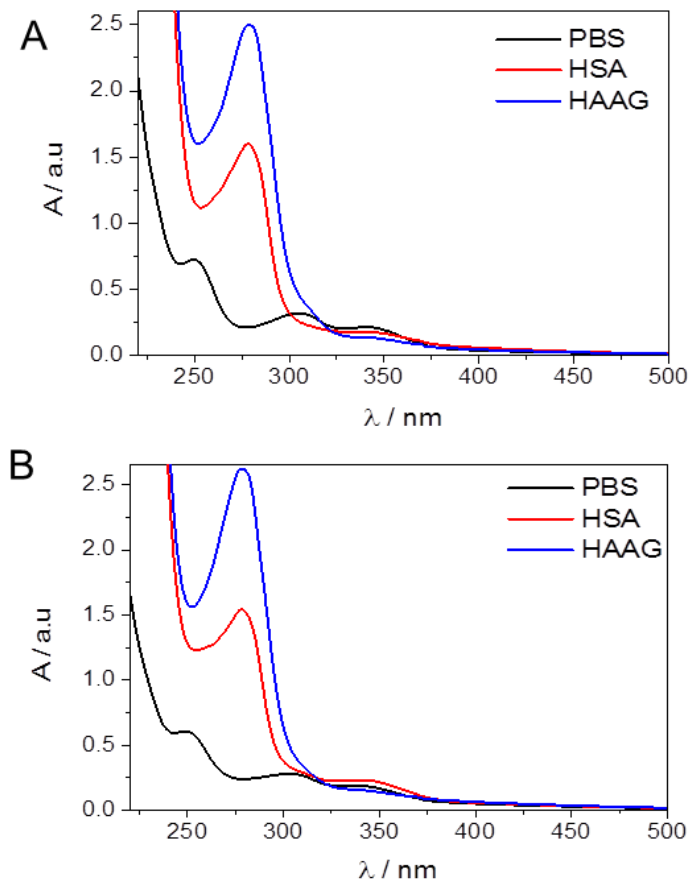

Figure 1. UV absorption spectra of A: MMP and $\mathbf{B}$ : MPA in different media: PBS (black), HSA at 1:1 ligand@protein molar ratio (red) and HAAG at 1:2 ligand@protein molar ratio (blue). Concentration of ligand was $4.0 \times 10^{-5} \mathrm{M}$.

A

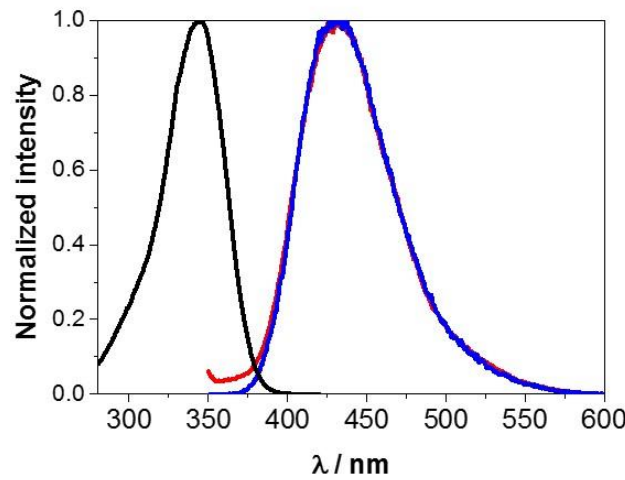

B

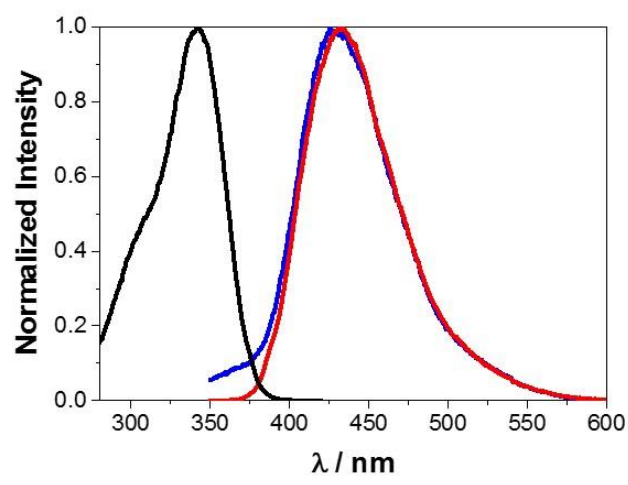

Figure 2. Fluorescence spectra at $\lambda_{\mathrm{exc}}=340 \mathrm{~nm}$ (blue) and at $\lambda_{\mathrm{exc}}=310$ (red); and excitation spectra at $\lambda_{\mathrm{em}} 432 \mathrm{~nm}$ (black) of A: MMP and B: MPA $\left(6.0 \times 10^{-5} \mathrm{M}\right.$ in PBS). 
The fluorescence quantum yields $\left(\phi_{\mathrm{F}}\right)$ of MMP and MPA were determined using carbazole as standard [12], the corresponding values are presented in Table 1, entries 1 and 4; both were lower than 0.1 , although $\phi_{\mathrm{F}}(\mathrm{MMP})$ was somewhat higher than $\phi_{\mathrm{F}}$ (MPA). The fluorescence lifetimes $\left(\tau_{\mathrm{F}}\right)$ were under the detection limit of our apparatus (lower than $1 \mathrm{~ns}$ ).

Table 1. Fluorescence quantum yields and lifetimes of MMP and MPA in different media.

\begin{tabular}{lllll}
\hline Entry & Compound $^{[\mathrm{a}]}$ & Medium $^{[\mathrm{b}]}$ & $\phi_{\mathrm{F}}{ }^{[\mathrm{c}]}$ & $\begin{array}{c}\tau_{\mathrm{F}}{ }^{[\mathrm{c}]} \\
(\mathrm{ns})\end{array}$ \\
\hline 1 & MMP & PBS & 0.095 & $<1$ \\
2 & MMP & $\mathrm{HSA}^{\mathrm{c}}$ & 0.220 & 5.7 \\
3 & MMP & $\mathrm{HAAG}^{\mathrm{c}}$ & 0.228 & 2.8 \\
4 & MPA & $\mathrm{PBS}^{\mathrm{c}}$ & 0.046 & $<1$ \\
5 & MPA & $\mathrm{HSA}^{\mathrm{c}}$ & 0.294 & 5.1 \\
\hline
\end{tabular}

${ }^{[\mathrm{a}]}[\mathrm{MMP}]=[\mathrm{MPA}]=3.33 \times 10^{-5} \mathrm{M} ;{ }^{[\mathrm{b}]}[\mathrm{HSA}]=3.33 \times 10^{-5} \mathrm{M} ;[\mathrm{HAAG}]=6.66 \times 10^{-5}$ $\mathrm{M} ;{ }^{\left[{ }^{[c]}\right.} \lambda_{\mathrm{exc}}=340 \mathrm{~nm}$; carbazole was used as standard ( $\phi_{\mathrm{F}}=0.42$, in $\left.\mathrm{EtOH}\right)$;

A

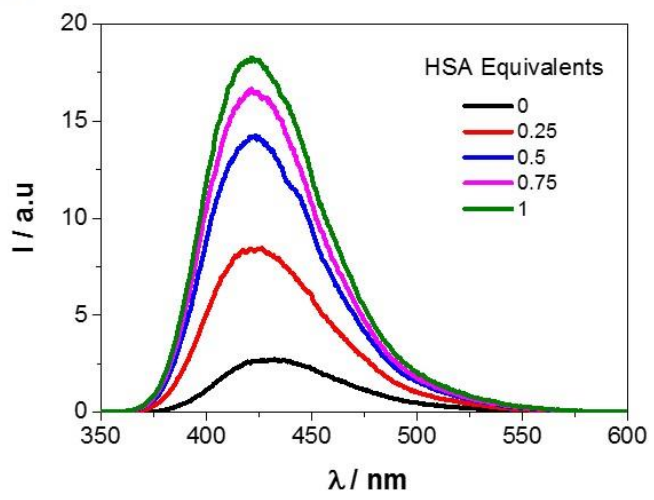

B

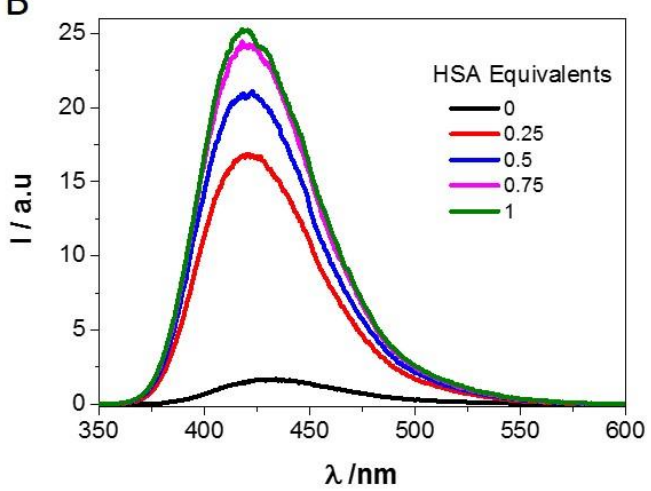

Figure 3. Fluorescence spectra $\left(\lambda_{\mathrm{exc}}=340 \mathrm{~nm}\right)$ in the presence of increasing amounts of HAS of MMP (A) and MPA (B) at $6.0 \times 10^{-5} \mathrm{M}$.

\section{Photophysical properties of MMP and MPA within HSA}

The fluorescence spectra of MMP and MPA in the presence of increasing amounts of HSA are shown in Figures 3A and 3B, respectively. For both MMP and MPA, addition of protein led to a hypsochromic shift of the band, with the new maximum located at $422 \mathrm{~nm}$; besides, the fluorescence intensity increased dramatically after HSA addition. These trends agree with an efficient ligand/protein interaction, as revealed further by the enhanced $\phi_{\mathrm{F}}$ and $\tau_{\mathrm{F}}$ values, determined at 1:1 ligand/protein molar ratio (Table 1, entries 2 and 5).

Fluorescence quantum yields were obtained using carbazole as standard $\left(\phi_{\mathrm{F}(\mathrm{std})}(\mathrm{EtOH})=0.42\right)[12]$, using eq. 1

$$
\phi_{F(i)}=\frac{A_{(i)}}{A_{s t d}} \cdot \frac{A b s_{(s t d)}}{A b s_{(i)}} \cdot \frac{n}{n_{s t d}} \cdot \phi_{F(s t d)}
$$

eq. 1

where, $A_{(i)}$ is the fluorescence area of the drugs, $A_{s t d}$ is the fluorescence area of standard, $A b s$ and $A b s_{(s t d)}$ corresponds to the absorbance intensity at $340 \mathrm{~nm}$ of drug and standard, respectively, and $n$ is the refraction index of solvent employed.

The binding constants $\left(\mathrm{K}_{\mathrm{B}}\right)$ for MMP@HSA and MPA@HSA complexes were determined using the modified BenesiHildebrand equation [13], where the stoichiometry of the complex was taken as 1, according to the Job-plot curves (Figure S1 in Supporting Information) [14]

$$
\frac{1}{F-F_{0}}=\frac{1}{\left(F_{\max }-F_{\min }\right)}+\frac{1}{K_{B}\left(F_{\max }-F_{0}\right)} \cdot \frac{1}{C^{n}}
$$

eq. 2

where, $F$ corresponds to the fluorescence intensity of the drugs at each protein concentration $\left(0\right.$ to $\left.6.0 \times 10^{-5} \mathrm{M}\right), F_{0}$ is the fluorescence of the MMP or MPA in the absence of proteins, $C$ is the molar protein concentration and $n$ is the stoichiometry of the complex, determined by the Job-plot graphs ( 1 in all cases).

Thus, from the plot of $1 /\left(\mathrm{I}-\mathrm{I}_{\mathrm{O}}\right)$ vs $1 /$ [HSA] (Figure 4), $\mathrm{K}_{\mathrm{B}}$ values of $3.2 \times 10^{4} \mathrm{M}^{-1}(\mathrm{MMP})$ and $1.3 \times 10^{5} \mathrm{M}^{-1}$ (MPA) were obtained, indicating a moderate-strong drug/protein interaction, somewhat smaller than that of warfarin $\left(\mathrm{K}_{\mathrm{B}}=3.3 \times 10^{5} \mathrm{M}^{-1}\right)$ but higher than antihistaminic epinastine hydrochloride $\left(\mathrm{K}_{\mathrm{B}}=2.72 \times\right.$ $\left.10^{4} \mathrm{M}^{-1}\right)$, the antiallergic cetirizine hydrochloride $\left(\mathrm{K}_{\mathrm{B}}=2.4 \times 10^{4}\right.$ $\left.\mathrm{M}^{-1}\right)$ [15].

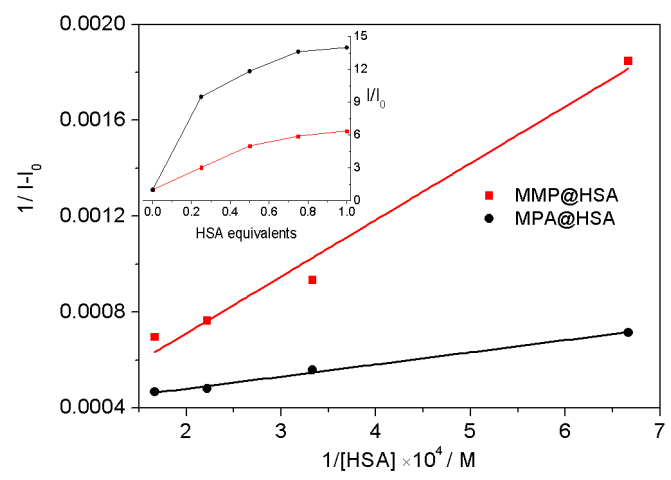

Figure 4. Benesi-Hildebrant plot for MMP@HSA and MPA@HSA. Inset: Changes in the fluorescence intensity of MMP and MPA with increasing concentration of HSA. $[\mathrm{MMP}]=[\mathrm{MPA}]=6.0 \times 10^{-5} \mathrm{M}$. 
In order to assess the nature of the binding site of MMP and MPA within HSA, displacement experiments using oleic acid (OLE) and ibuprofen (IBU) as probes for site I and site II, respectively, were performed (Figures 5A and 5B) [16]. Thus, addition of OLE to a 1:1 mixture of MMP@HSA gave rise to an enhancement of the fluorescence intensity until 2 equivalents of probe added (Figure 5A). This can be explained by a simultaneous binding of OLE and MMP in different subsites of site I under these conditions [8a], resulting in an allosteric effect that provokes a compression of the site and a parallel fluorescence enhancement of MMP. Allosteric effects have been reported when the properties of a macromolecule change as a consequence of a ligand binding to this macromolecule, altering its affinity towards another ligand. This is attributed to conformational changes in the protein cavity and the effect can be monitored by different techniques, such as fluorescence, surface plasmon resonance or cyclic voltammetry. [17] Addition of higher amounts of OLE, from 3 to 5 equivalents, led to a progressive decrease of the fluorescence intensity, indicating that under these conditions MMP is being expelled from the intraprotein region to the bulk solution. These results point to site I as the main binding site for MMP within HSA. Accordingly, addition of IBU to the MMP@HSA complex did not result in significant changes of the fluorescence spectra (Figure 5A, Inset), indicating poor affinity of MMP by site II. The same trend was observed for MPA (Figure 5B). Remarkably, this selective UV-A excitation of the drug avoids misleading effects due to protein absorption.

A

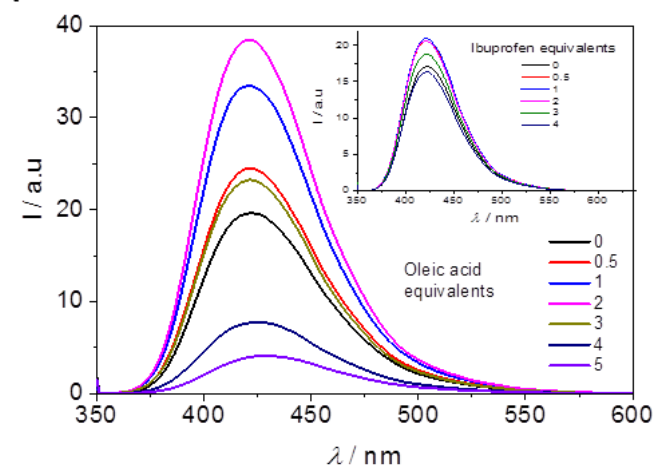

B

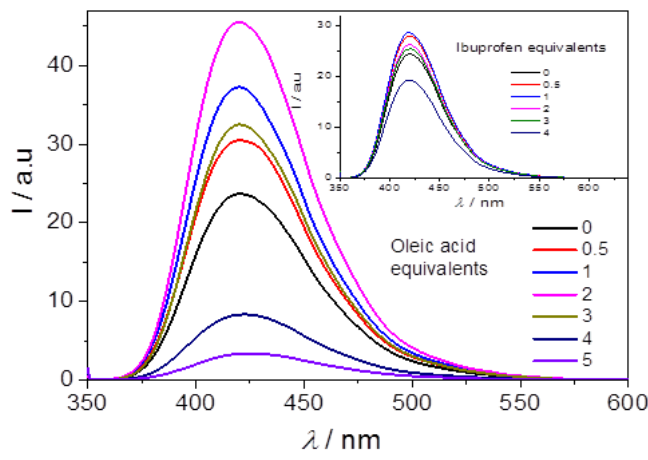

Figure 5. Displacement experiments on MMP@HSA and MPA@HSA. Fluorescence spectra of A: MMP@HSA + oleic acid; Inset: MMP@HSA + Ibuprofen; B: MPA@HSA + oleic acid; Inset: MPAHSA + ibuprofen. $[\mathrm{MMP}]=3.33 \times 10^{-5} \mathrm{M} ;[\mathrm{MPA}]=4.0 \times 10^{-5}$ M.

\section{Photophysical properties of MMP and MPA in HAAG}

A parallel set of experiments were performed using HAAG. Thus, the fluorescence spectra of MMP and MPA were recorded after addition of different amounts of protein are (Figure 6). Contrarily to that observed in the experiments with HSA, the behavior of prodrug and drug was diverging in the presence of HAAG. Thus, while addition of increasing amounts of HAAG (until 5 equivalents) to MMP led to a dramatic enhancement of the fluorescence intensity, concomitant with a shift of the maxima towards shorter wavelengths (from $\lambda=432$ to $422 \mathrm{~nm}$ ), the changes for MPA were almost negligible. This indicates very poor binding of MPA to HAAG. Again, $\mathrm{K}_{\mathrm{B}}$ was determined using the modified Benesi-Hildebrand equation [13] (taking into account a 1:1 stoichiometry for the complex, determined from the Job plot, shown in the Supporting information) [14]. Thus, $K_{B}$ was determined as $1.1 \times 10^{4} \mathrm{M}^{-1}$.

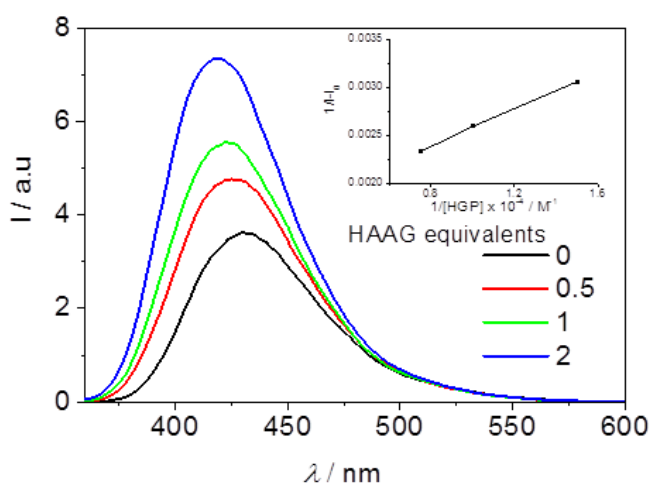

Figure 6. Fluorescence spectra $\left(\lambda_{\text {exc }}=343 \mathrm{~nm}\right)$ in the presence of increasing amounts of HAAG. $[\mathrm{MMP}]=3.33 \times 10^{-5} \mathrm{M}$. Inset: Benesi-Hildebrant Plot for MMP@2HAAG.

\section{Computational studies}

In an effort to gain further insight into the binding interactions responsible for the affinity obtained, the binding modes of compounds MPA and MMP with HSA and HAAG were studied. Molecular docking using GOLD 5.2.2 [18] program and the protein coordinates found in the crystal structure of HSA in complex with palmitic acid (PDB code 4BKE, $2.35 \AA$ A ) [19] and HAAG in complex with chlorpromazine (PDB code 3APX, 2.2 $\AA$ ) [20] was performed. These structures were chosen because: (a) 4BKE contains a fatty acid (palmitic acid) molecule in site I and has higher resolution than the rest of the available HSA/fatty acid crystallographic structures and (b) the ligand observed intertiary nitrogen as MMP.

Binding mode of MPA and MMP with HSA - Considering that MPA binds to HSA site I and that can be replaced with fatty acids, sub-domain IIA, in which a palmitic acid molecule is observed, was selected as binding pocket. Our docking studies showed that MPA would be fixed to sub-domain IIA by three strong contacts. Thus, it would have electrostatic interactions between its carboxylate moiety and the guanidinium groups of $\operatorname{Arg} 222$ and Arg218, which are located close to Trp214, and a hydrogen bond interaction between its phenol group and the side chain of Arg257 (Figures 7A and 7D). In addition, MPA would also have numerous aliphatic interactions with the apolar residues $3 \mathrm{APX}$ has also an aromatic moiety and a long chain with a within the pocket, specifically, residues Ala291, Leu238, and Cys253/Cys245 disulfide bridge, as well as a $\pi-\pi$ stacking interaction between its aromatic moiety and His242 (Figure S3). 
A

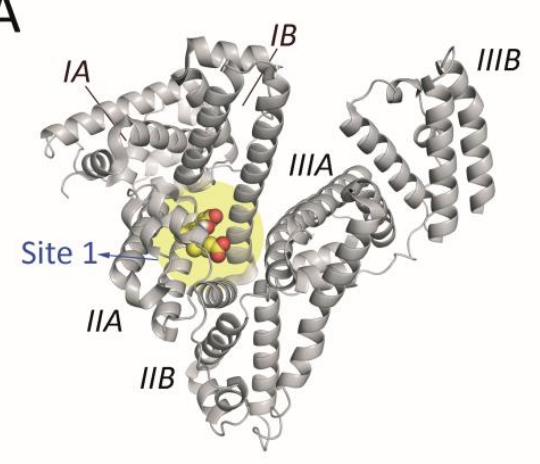

B

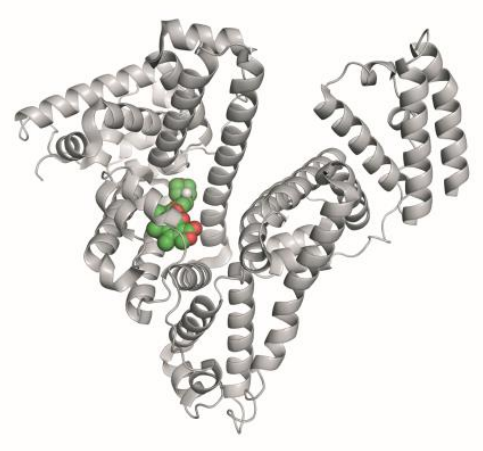

C

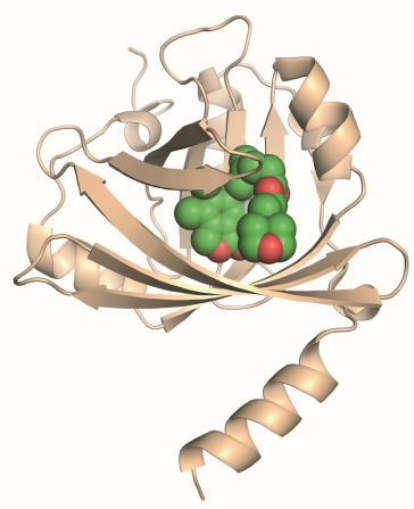

D

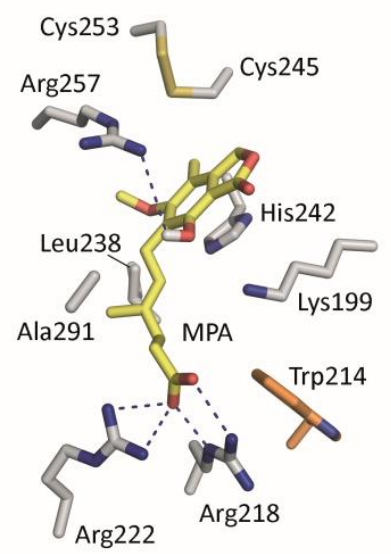

E

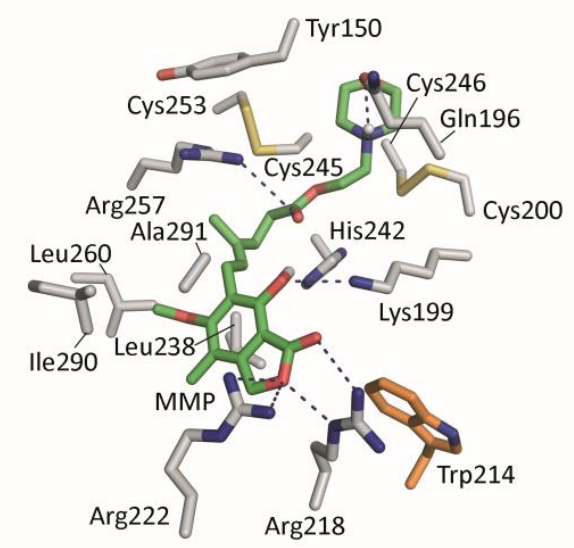

F

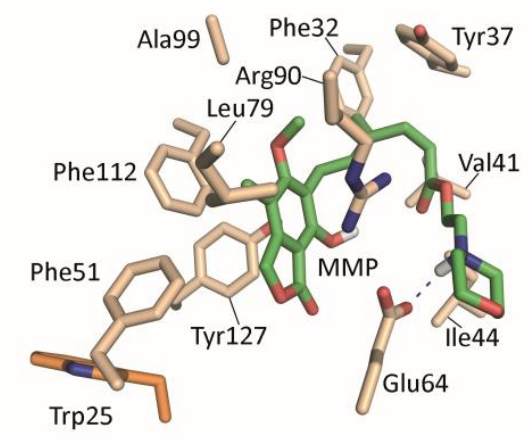

Figure 7. Predicted binding mode of MPA and MMP. (A-C) Overall view of the HSA/MPA (A), HSA/MMP (B) and HAAG/MMP (C) complexes. The main backbone of MPA and MMP are shown as yellow and green spheres, respectively. The domains I-III and sub-domains (A,B) in HSA protein are labelled. (D-E) Relevant interactions of MPA (D) and MMP (E) with sub-domain IIA (site 1) of HSA. (F) Interactions of MMP with HAAG. Trp214 side chain is highlighted in orange. Note how in all cases Trp214 (yellow) is close by the ligands. Hydrogen bonding and electrostatic interactions between the ligand and the proteins are shown as blue dashed lines. Relevant side chain residues are shown and labelled.

As expected for a non negatively charged ligand, the binding mode of MMP to sub-domain IIA was predicted to be significantly different from that of MPA (Figures 7B and 7E). As a matter of fact, since the residues located close to Trp214 are positively charged, the aromatic ring of MMP is now located very close to Trp214 whereas the side chain containing the morpholine (positively charged) and ester moieties are placed at the other end of the sub-domain IIA. The ligand would be anchored to this sub-domain by hydrogen bonding interactions with residues $\operatorname{Arg} 222, \operatorname{Arg} 218, \operatorname{Arg} 257$ and Gln196. As for MPA, the ligand is well surrounded by a series of attractive apolar interactions with residues Leu260, Ile290, Ala291, Leu238, Tyr150, Cys200/Cys246 and Cys253/Cys245 disulfide bridges (Figure S3). Comparison of the binding mode of the two ligands revealed that the aromatic moiety of MMP would be located closer to Trp214 than the corresponding one in MPA.

Binding mode of MMP with HAAG - The recognition center of HAAG is a quite spherical apolar cavity, whose entrance is flanked by mainly two negatively charged residues (Glu92 and Glu64). This arrangement disfavors the binding of negatively charged ligands as MPA, as experimentally observed. Our docking studies revealed that MMP would be soaked into this large apolar pocket by numerous lipophilic interactions with the residues within the pocket (Phe32, Tyr37, Val41, Ile44, Phe51, Leu79, Ala99, Phe112 and Tyr127) (Figures 7C and 7F). Moreover, the flexible side chain would be anchored into the entrance of the pocket by a strong electrostatic interaction between the morpholine moiety and the carboxylate group of Glu64. As for HSA/MMP complex, the aromatic moiety of the ligand would be located close to the only tryptophan residue of the protein, Trp25.

\section{Conclusions}

The intrinsic emission properties of MMP and MPA upon excitation of their long-wavelength absorption maxima in the UVA exhibit remarkable changes upon binding with transport proteins. Thus, the fluorescence quantum yields and lifetimes increase markedly and the emission maxima undergo a significant hypsochromic shift. Displacement experiments with selective probes allow determination of the high affinity binding site in HSA, which turns out to be Sudlow site I for both for the drug and its metabolite. As regards HAAG, it is able to bind selectively MMP, but not MPA. Docking studies revealed significant molecular binding differences in the binding of MMP and MPA to sub-domain IIA of HSA (site 1). Thus, for MPA, the aromatic moiety would be in close contact to Trp214 with the flexible chain pointing to the other end of the sub-domain. On the contrary, for MMP, the carboxylate group of the chain would be fixed nearby Trp214 through electrostatic interactions with residues Arg218 and Arg222. Moreover, the architecture of the recognition center of HAAG, which is a spherical cavity with an entrance flanked by two negatively charged residues, explains the absence of binding with MPA as experimentally observed. For MMP, the strong electrostatic interaction between the morpholine moiety and the carboxylate group of Glu64 and the numerous 
lipophilic interactions with the deep spherical apolar cavity would be responsible for its effective binding to HAAG.

\section{Experimental section}

Chemicals. Mycophenolate mofetil (MMF), mycophenolic acid (MPA), Human serum albumin (HSA), and human $\alpha 1$-acid glycoprotein (HAAG) were purchased from Sigma-Aldrich. Solutions of phosphate buffer saline (PBS, $0.01 \mathrm{M}, \mathrm{pH}$ 7.4) were obtained dissolving Phosphate-buffered saline tablets (SigmaAldrich) in Milli-Q water. Stock solutions of the drugs were prepared at the concentration of $1 \mathrm{mM}$ in acetone and then were stored at $4{ }^{\circ} \mathrm{C}$. To prepare the samples, the acetone was evaporated at room temperature and dissolved then in PBS. Solutions containing drugs and proteins were prepared and kept overnight at $4{ }^{\circ} \mathrm{C}$ and then were tempered at room temperature before measurements.

Absorption measurements. Steady state absorption spectra were recorded in a JASCO V-630 spectrophotometer. The stoichiometry of MMP@HSA, MPA@HSA and MMP@HAAG complexes was determined by following the absorbance changes of MMP or MPA at $340 \mathrm{~nm}$ when the molar fraction of protein changes from 0 to 1 . The total concentration (MMP or MPA + protein) was constant $(0.122 \mathrm{mM})$. For determination of MMP@HAAG stoichiometry, the total concentration was $4 \times 10^{-}$ $5 \mathrm{M}$ and the absorbance changes in the UV spectra were monitored at $313 \mathrm{~nm}$.

Fluorescence experiments. The emission spectra were recorded on a JASCO FP-8500 spectrofluorimeter system, provided with a monochromator in the wavelength range 200850. Absorbance of MMP and MPA was 0.1 at $\lambda_{\mathrm{exc}}=340 \mathrm{~nm}$. Quartz cells with $1 \mathrm{~cm}$ path length and $3 \mathrm{ml}$ of capacity were employed. For the experiments in the presence of protein, concentration of MMP and MPA was maintained constant $(6.0 \times$ $10^{-5} \mathrm{M}$ and the HSA equivalents added ranged from 0.25 to 1 .

Site selective experiments were performed using oleic acid and ibuprofen as probes for site I and site II, respectively. ${ }^{15}$ Micromolar amounts of a oleic acid stock solution in acetonitrile $(24 \mathrm{mM})$ were added $(0-5$ equivalents) to the cuvette containing MMP or MPA and HSA in equimolar ratio. Concentrations of MMP and MPA were $3.33 \times 10^{-5} \mathrm{M}$ and $4.0 \times 10^{-5} \mathrm{M}$, respectively. Parallel experiments were done by adding pure acetonitrile, in order to exclude possible interferences. In all cases, $15 \mathrm{~min}$ of incubation was taken before performing the measurements to ensure stabilization.

In case of ibuprofen, in PBS solution that contained MMF $\left(3.33 \times 10^{-5} \mathrm{M}\right)$ or MPA $\left(4.0 \times 10^{-5} \mathrm{M}\right)$ and protein $(1: 1)$ different equivalents of the site probe were added (from 0.5 to 4 ). The fluorescence intensity was measured in each cuvette.

Fluorescence lifetimes were recorded on a PTI (Photon Technology International) fluorimeter. It contains a pulsed LED excitation source, a sample compartment, and a lifetime detector. The software used to lifetime analysis was EasyLife X. The source LEDs employed were $340 \mathrm{~nm}$ with a 370 emission filter.

Docking studies. They were carried out using program GOLD 5.2.2 [18] and the protein coordinates found in the crystal structure of HAAG in complex with chlorpromazine (PDB code 3APX, $2.2 \AA$ ) [19] and of HSA in complex with palmitic acid (PDB code 4BKE, $2.35 \AA$ ) [20]. Ligand geometries were minimized using the AM1 Hamiltonian as implemented in the program Gaussian 09 [21] and used as MOL2 files. Each ligand was docked in 25 independent genetic algorithm (GA) runs, and for each of these a maximum number of $100000 \mathrm{GA}$ operations were performed on a single population of 50 individuals. Operator weights for crossover, mutation and migration in the entry box were used as default parameters $(95,95$, and 10 , respectively), as well as the hydrogen bonding (4.0 $)$ ) and van der Waals $(2.5 \AA)$ parameters. The position of: (i) chlorpromazine present in the crystal structure of HAAG (PDB 3APX) and (ii) palmitic acid PLM1007 in the crystal structure of HSA (PDB 4BKE) was used to define the active-site and the radius was set to $6 \AA$. All crystallographic water molecules and the aforementioned ligands were removed for docking. The "flip ring corners" flag was switched on, while all the other flags were off. The GOLD scoring function was used to rank the ligands in order to fitness. The molecular graphics program PyMOL [22] was employed for visualization and depicting ligand/protein structures.

\section{Acknowledgments}

Financial support from the Spanish Ministry of Economy and Competiveness (CTQ2013-47872-C2-1-P, CTQ2016-78875-P, SAF2016-75638-R), Xunta de Galicia, (Centro singular de investigación de Galicia accreditation 2016-2019, ED431G/09), the European Regional Development Fund (ERDF) and the Generalitat Valenciana (PROMETEO/2017/075) is gratefully acknowledged.

\section{References}

1. (a) T. Bohnert, L.-S. Gan Li, Plasma protein binding: from discovery to development, J. Pharm. Sci. 102 (2013) 2953-2994; (b) P. Keen, "Effect of Binding to Plasma Proteins on the Distribution, Activity and Elimination of Drugs", In: Concepts in Biochemical Pharmacology. Handbook of Experimental Pharmacology, (Eds: Brodie B.B., Gillette J.R., Ackerman H.S), Springer, Berlin, Heidelberg, (1971), vol 28/1, pp. 213-231; (c) A. Dennis, L.D. Smith, E.H. Kerns, The effect of plasma protein binding on in vivo efficacy: misconceptions in drug discovery, Nature Rev. Drug Discov. 9 (2010), 929-939; (d) N. van Brandt, P. Hantson, "Pharmacokinetics and Drug-Protein Binding", In: Yearbook of Intensive Care and Emergency Medicine, (Ed.: J. L. Vincent), Springer, Berlin, Heidelberg, (1996), Vol 2, pp. 761-770; (d) A. Yasgar, S.D. Furdas, D.J. Maloney, A. Jadhav, M. Jung, A. Simeonov, PLoS One 7 (2012), e45594

2. (a) T. Peters, "Serum Albumin", In: Advances in Protein Chemistry, Academic Press, New York, (1985), Vol 37, pp. 161-245; (b) X.U. Kragh-Hansen, V.T.G. Chuang, M. Otagiri, Practical aspects of the ligand-binding and enzymatic properties of human serum albumin, Biol. Pharm. Bull. 25 (2002), 695-704; (c) M. Fasano, S. Curry, E. Terreno, M. Galliano, G. Fanali, P. Narciso, S. Notari, P. Ascenzi, The extraordinary ligand binding properties of human serum albumin, IUBMB Life 57 (2005), 787-796; (d) Z.H. Israili, P.G. Dayton, Human alpha-1-glycoprotein and its interactions with drugs, Drug Metab. Rev. 33 (2001), 161-235; (e) T. Fournier, N. Medjoubi-N, D. Porquet, Alpha-1-acid glycoprotein, Biochim. Biophys. Acta 1482 (2000), 157-171.

3. (a) S. Sugio, A. Kashima, S. Mochizuki, M. Noda, K. Kobayashi, Crystal structure of human serum albumin at 2.5 A resolution, Protein Eng. 12 (1999), 439-446; (b) M. Dockal, D.C. Carter, F. Rüker, The three recombinant domains of human serum albumin. Structural characterization and ligand binding properties, J. Biol. Chem. 274 (1999), 29303-29310; (c) X.M. He, D.C. Carter, Atomic structure and chemistry of human serum albumin, Nature 358 (1992), 209-215.

4. (a) V. Kopecký, R. Ettrich, K. Hofbauerová, V. Baumruk, Structure of human alpha1-acid glycoprotein and its high-affinity binding site, Biochem. Biophys. Res. Commun. 300 (2003), 41-46; (b) K. Schmid, " $\alpha 1$ acid Glicoprotein", In: The Plasma proteíns: structure, function and genetic control, Ed: Putnam F. W, Academic Press, New York, (1975), Vol 1; (c) K. Schmid, H. Kaufmann, S. Isemura, F. Bauer, J. Emura, T. Motoyama, M. Ishiguro, S. Nanno, Structure of $\alpha 1$-acid glycoprotein. The complete amino acid sequence, multiple amino acid substitutions, and homology with the immunoglobulins, Biochemistry 12 (1973), 2711-2724. 
5. (a) Z.D. Zhivkova, Studies on drug-human serum albumin binding: the current state of the matter, Curr. Pharm. Des. 21 (2015), 1817-1830; (b) J. Ghuma, P.A. Zunszain, I. Petitpas, A. A. Bhattacharya, M Otagiri, S. Curry, Structural basis of the drug-binding specificity of human serum albumin, J. Mol. Biol. 353 (2005), 38-52; (c) K.M. Wasan, D.R. Brocks, S.D. Lee, K. Sachs-Barrable, S. J. Thornton, Impact of lipoproteins on the biological activity and disposition of hydrophobic drugs: implications for drug discovery, Nat. Rev. Drug Discov. 7 (2008), 84-99.

6. A.C. Allison, E.M. Eugui, Mycophenolate mofetil and its mechanisms of action, Immunopharmacology 47 (2000), 85-118.

7. J.E. Kitchin, M.K. Pomeranz, G. Pak, K. Washenik, J.L. Shupack, Rediscovering mycophenolic acid: a review of its mechanism, side effects, and potential uses, J. Am. Acad. Dermatol. 37 (1997), 445449.

8. (a) X. Ma, J. Yan, Q. Wang, D. Wu, H. Li, Spectroscopy study and coadministration effect on the interaction of mycophenolic acid and human serum albumin, Int. J. Biol. Macromol. 77 (2015), 280-286; (b) N.T. Vethe, U.E. Kongsgaard, A.M. Andersen, P.D. Line, A.M. Ali, S. Bergan, Mycophenolate pharmacokinetics and inosine monophosphate dehydrogenase activity in liver transplant recipients with an emphasis on therapeutic drug monitoring, Scand. J. Clin. Lab. Invest. 73 (2013), 117-124; (c) M. Qasim, H. Rahman, M. Oellerich, A.R. Asif, Fetal calf serum heat inactivation and lipopolysaccharide contamination influence the human $\mathrm{T}$ lymphoblast proteome and phosphoproteome, Prot. Sci. 9 (2011), 57-69; (d) M.A. Navia, Protein-drug complexes important for immunoregulation and organ transplantation, Curr. Opin. Struct. Biol. 6 (1996), 838-847.

9. B.A. Atcheson, P.J. Taylor, D.W. Mudge, D.W. Johnson, C.M Hawley, S.B, Campbell, M. Isabel Nixcole, P.I. Pillians, S.E Tett, Chlorpropamide 2-hydroxylation is catalysed by CYP2C9 and CYP2C19 in vitro: chlorpropamide disposition is influenced by CYP2C9, but not by CYP2C19 genetic polymorphism, Br. J. Clin. Pharmacol. 59 (2005), 271-280.

10. Y. Vial, M. Tod, M. Hornecker, S. Urien, F. Conti, A. Dauphin, Y. Calmus, B. Blanchet, In vitro influence of fatty acids and bilirubin on binding of mycophenolic acid to human serum albumin, J. Pharm. Biomed. Anal. 54 (2011), 607-609.

11. L. Khalafi, M. Rafiee, F. Mahdiuna, S. Sedaghata, Investigation of the inclusion complex of $\beta$-cyclodextrin wit mycophenolate moSpectrochim. Acta A 90 (2012), 45-49.

12. M. Montalti, A. Credi, L. Prodi, M. Gandolfi, in Handbook of photochemistry. 3rd edition, Tailor \& Francis, CRC Press, Boca Raton, (2006), pp. 572-575.

13. A.A. Elabd, A new fluorescent sensor for determination of thorium by thin film of 2-(acetyloxy)- $N$-(5-nitro-2-thiazolyl)-benzamide embedded in sol-gel matrix, RSC Adv., 6 (2016), 45525-45532.

14. C.Y. Huang, Determination of binding stoichiometry by the continuous variation method: the Job plot, Methods Enzymol. 87 (1982), 509-525.

15. T. C. Pinkerton, K. A. Koeplinger, Determination of warfarin-human serum albumin binding parameters by an improved Hummel-Dreyer high performance liquid chromatographic method using internal surface reversed-phase columns, Anal. Chem. 62 1(990), 2114-2122.

16. G. Sudlow, D.J. Birkett, D.N. Wade, Further characterization of specific drug binding sites on human serum albumin, Mol. Pharmacol. 12 (1976), 1052-1061.
17. (a) D.E. Epps, T.J. Raub, F.J. Kezdy, A general, wide-rage spectrofluorometric method for measuring the site-specific affinities of drugs toward human serum albumin, Anal. Biochem. 227 (1995), 342350; (b) M. Marin, V. Lhiaubet-Vallet, M.A. Miranda, Site-dependent photo-fries rearrangement within serum albumins, J. Phys. Chem. B 115 (2011), 2910-2915; (c) R. Pérez-Ruiz, E. Lence, I. Andreu, D. Limones-Herrero, C. González-Bello, M.A. Miranda, M.C. Jiménez, A new pathway for protein haptenation by $\beta$-lactams, Chem. Eur. J. 23 (2017), 13986-13994; (d) C. Yan-Min, G. Lianf-Hong, Combined fluorescence and electrochemical investigation on the binding interaction between organic acid and human serum albumin, J. Environ. Sc. 21 (2009), 373-379; (e) Y. Kuroda, M. Saito, H. Sakai, T. Yamaoka, Rapid characterization of Drug-drug interaction in Plasma Protein Binding using a Surface Plasmone Resonance Biosensor, Drug Metab. Pharmacokinet. 23 (2008), 120-127; (f) Z. Mai, X. Zhao, Z. Dai, X. Zou, Investigation on the chloride effect on hemoglobin by absortive transfer voltammetry, Anal. Biochem. 399 (2010) 23-29.

18. https://www.ccdc.cam.ac.uk/solutions/csd-discovery/components/gold/

19. A. Sivertsen, J. Isaksson, H.S. Leiros, J. Svenson, J. Svendsen, B. O. Brandsdal, Synthetic cationic antimicrobial peptides bind with their hydrophobic parts to drug site II of human serum albumin, BMC Struct. Biol. (2014), 14:4 doi: 10.1186/1472-6807-14-4.

20. Crystal structure deposited in the Protein Data Bank by K. Nishi, T. Ono, T. Nakamura, N. Fukunaga, M. Izumi, H. Watanabe, A. Suenaga, T. Maruyama, Y. Yamagata, S. Curry, M. Otagiri.

21. M. J. Frisch, G. W. Trucks, H. B. Schlegel, G. E. Scuseria, M. A. Robb, J. R. Cheeseman, G. Scalmani, V. Barone, B. Mennucci, G. A. Petersson, H. Nakatsuji, M. Caricato, X. Li, H. P. Hratchian, A. F. Izmaylov, J. Bloino, G. Zheng, J. L. Sonnenberg, M. Hada, M. Ehara, K. Toyota, R. Fukuda, J. Hasegawa, M. Ishida, T. Nakajima, Y. Honda, O. Kitao, H. Nakai, T. Vreven, J. A. Montgomery, Jr., J. E. Peralta, F. Ogliaro, M. Bearpark, J. J. Heyd, E. Brothers, K. N. Kudin, V. N. Staroverov, R. Kobayashi, J. Normand, K. Raghavachari, A. Rendell, J. C. Burant, S. S. Iyengar, J. Tomasi, M. Cossi, N. Rega, J. M. Millam, M. Klene, J. E. Knox, J. B. Cross, V. Bakken, C. Adamo, J. Jaramillo, R. Gomperts, R. E. Stratmann, O. Yazyev, A. J. Austin, R. Cammi, C. Pomelli, J. W. Ochterski, R. L. Martin, K. Morokuma, V. G. Zakrzewski, G. A. Voth, P. Salvador, J. J. Dannenberg, S. Dapprich, A. D. Daniels, Ö. Farkas, J. B. Foresman, J. V. Ortiz, J. Cioslowski, and D. J. Fox, Gaussian 09, Revision D.01, Gaussian, Inc.: Wallingford CT, 2009.

22. http://www.pymol.org

\section{Supplementary Material}

Determination of the stoichiometry of ligand@protein complexes by means of the Job Plot. Detailed view of the proposed binding mode of MPA with HSA and MMP with HSA and HAAG.

Click here to remove instruction text.. 